\title{
Thrombotic thrombocytopenic purpura as a rare cause of anemia with thrombocytopenia in childhood: report of 2 cases
}

\author{
Oya Köker ${ }^{1}$, Zeynep Yıldız Yıldırmak², Dildar Bahar Genç², Önder Kılıçaslan \\ ${ }^{2}$ Department of Pediatric Hematology and Oncology and ${ }^{1}$ Department of Pediatrics, Şişli Hamidiye Etfal Training and \\ Research Hospital, Istanbul, Turkey. E-mail: oyakoker@hotmail.com \\ Received: 21st November 2017, Revised: 4th March 2018, Accepted: 15th April 2018
}

\begin{abstract}
SUMMARY: Köker O, Yıldırmak ZY, Genç DB, Kılıçaslan Ö. Thrombotic thrombocytopenic purpura as a rare cause of anemia with thrombocytopenia in childhood: report of 2 cases. Turk J Pediatr 2019; 61: 418-423.

Thrombotic thrombocytopenic purpura (TTP) is a rare multisystem disorder characterized by single or recurrent episodes of thrombocytopenia, microangiopathic hemolytic anemia and widespread microvascular thrombosis, which causes significant morbidity and mortality unless promptly recognized and treated. The underlying pathogenesis is a defect in von Willebrand factor (vWF) cleaving protease, called "A Disintegrin and Metalloproteinase with Thrombospondin Type 1 Repeats 13 (ADAMTS-13)". There are 2 forms: congenital TTP (ADAMTS-13 gene mutations) and acquired TTP (autoantibodies and ADAMTS-13 deficiency). We presented two patients who initially presented with thrombotic microangiopathy and were later diagnosed with TTP upon demonstration of the deficiency in ADAMTS-13 activity.
\end{abstract}

Key words: ADAMTS-13, microangiopathy, Thrombotic thrombocytopenic purpura.

Thrombotic thrombocytopenic purpura (TTP) is a rare multisystem disorder characterized by single or recurrent episodes of thrombocytopenia, microangiopathic hemolytic anemia and widespread microvascular thrombosis, and may lead to ischemic multiorgan damage. ${ }^{1,2}$ It is associated with a deficiency in the activity of "A Disintegrin and Metalloproteinase with Thrombospondin Type 1 Repeats 13" (ADAMTS-13)", a metalloproteinase which cleaves ultra large Von Willebrand Factor multimers into smaller protein fragments. There are 2 forms of the disease, congenital TTP (ADAMTS-13 gene mutations) and acquired TTP (anti-ADAMTS-13 autoantibodies and ADAMTS-13 deficiency). Congenital TTP (Upshaw-Schulman Syndrome), generally occurs in early childhood, presenting with different clinical pictures ranging from asymptomatic thrombocytopenia episodes to multiorgan failure and sudden death. ${ }^{3}$ We presented two patients who initially presented with thrombotic microangiopathy and were later diagnosed with TTP upon demonstration of the deficiency in ADAMTS-13 activity. The aim of this report is to call attention to this entity which, despite being rare, may lead to high morbidity and mortality.

\section{Case 1}

A 12 year old boy was referred to our clinic with " bruising on his body and legs". There were no problems related to past medical history, nor a traumatic condition. Family history did not reveal any consanguinity or history of hematologic diseases.

On admission, the patient was in a poor general condition with pallor and tachycardia. The sclerae were icteric. The liver and spleen were not palpable on abdominal examination.

Laboratory examination revealed the following: Hemoglobin ( $\mathrm{Hb}): 7.4 \mathrm{~g} / \mathrm{dL}$, hematocrit: $21.3 \%$, Mean corpuscular volume (MCV): $106.9 \mathrm{fl}$, red blood cell count (RBC): $2.2 \mathrm{M} / \mathrm{L}$, 
RDW: 20.8 , reticulocytes: $14.9 \%$ and platelet count: $6.000 / \mathrm{mm}^{3}$.

The patient was further investigated for anemia and thrombocytopenia. A peripheral smear showed poikilocytosis, anisocytosis, presence of spherocytes, and $10 \%$ normoblasts. Biochemical studies revealed the following: Blood urea nitrogen (BUN): $68 \mathrm{mg} / \mathrm{dL}$, creatinine: $0.7 \mathrm{mg} / \mathrm{dL}$, Lactate dehydrogenase (LDH): $3380 \mathrm{U} / \mathrm{L}$, total bilirubin: $4.4 \mathrm{~g} / \mathrm{dL}$, direct bilirubin: $0.74 \mathrm{~g} / \mathrm{dL}$. The liver enzymes, electrolytes, urinalysis and coagulation tests were normal, and D-dimer was $1617 \mathrm{ng} /$ $\mathrm{mL}$. Unconjugated hyperbilirubinemia and a high LDH level also supported the findings of hemolysis.

Evans syndrome where autoimmune hemolytic anemia and autoimmune thrombocytopenia were both present, was suspected. However, this assumption was ruled out due to a negative direct Coombs test. Bone marrow aspiration, performed to rule out the conditions that infiltrate the bone marrow, showed prominent erythroid hyperplasia, whereas the megakaryocyte count was normal.

Anemia and thrombocytopenia persisted despite platelet and erythrocyte transfusions. On the $3^{\text {th }}$ day of his admission he developed emesis, dizziness, and agitation. Cranial computerized tomography findings were within normal limits. Microangiopathic hemoliytic anemia, thrombocytopenia and neurological findings indicated to the diagnosis of TTP. Plasmapheresis was initiated after blood samples were collected for ADAMTS-13. Pulse methylprednisolone treatment $\left(1000 \mathrm{mg} / 1.73 \mathrm{~m}^{2}\right.$ for 3 days) was given in combination with plasmapheresis and continued with daily $1 \mathrm{mg} / \mathrm{kg} /$ dose.

After the patient was treated with 7 sessions of plasmapheresis, the hemoglobine and platelet count did not improve significantly and the neurologic symptoms got worse with incomprehensible speech. Cranial imaging revealed hemorrhage in the right temporobasal area. Oxcarbazepine treatment was started. On week 3; he developed hypertension, proteinuria, and microscopic hematuria. Anti-dsDNA, C3 and C4 were normal. No signs of volume overload was noted. Calcium channel blocker was started as a treatment to control hypertension. An ADAMTS-13 level of $<2 \%$ and detectable anti-ADAMTS13 inhibitor levels confirmed the diagnosis of TTP. Plasmapheresis treatment along with metilprednisolon was continued.

Although the patient underwent 18 plasmaphereses during 1 months, no improvement was observed in terms of thrombocytopenia and anemia. He received platelet transfusion every other day because the platelet count was below 20.000, and erythrocyte transfusion every 5 days because the hemoglobin was under $7 \mathrm{~g} / \mathrm{dL}$. His neurologic symptoms and signs were progressively worsening including intermittent numbness of the extremities and face, speech disorder and seizures. On week 5 of the diagnosis, the patient died due to cerebral hemorrhage. The family was informed about the disease and their consent was taken to publish this case.

\section{Case 2}

A 14 month old boy with a complaint of coughing was referred to our hospital from a primary health care institution due to the presence of thrombocytopenia in his blood count, with a suspected diagnosis of idiopathic thrombocytopenic purpura or leukemia. Medical history of the patient revealed that there were no problems during delivery, nor any history of jaundice of the newborn period. He was an only child. There was no history of consanguinity or hematological diseases in the family medical history.

Physical examination detected poor general condition, a pale appearance, oropharyngeal hyperemia, serous nasal discharge and multiple insignificant lymphadenopathies in the cervical area. The patient had tachycardia and a 2/6 systolic murmur was heard in all foci. Abdominal palpation showed that the liver was palpable $4 \mathrm{~cm}$ below the right costal margin at the midclavicular line, and the spleen $2 \mathrm{~cm}$ below the left costal margin. Widespread petechial rashes were present only in the lower and upper extremities.

Laboratory examination revealed the following: Hb: $6.6 \mathrm{~g} / \mathrm{dL}$, hematocrit: $20.4 \%$, RBC: 2.7 M / L, MCV: 74.6 fl, RDW: 23.6\%, 


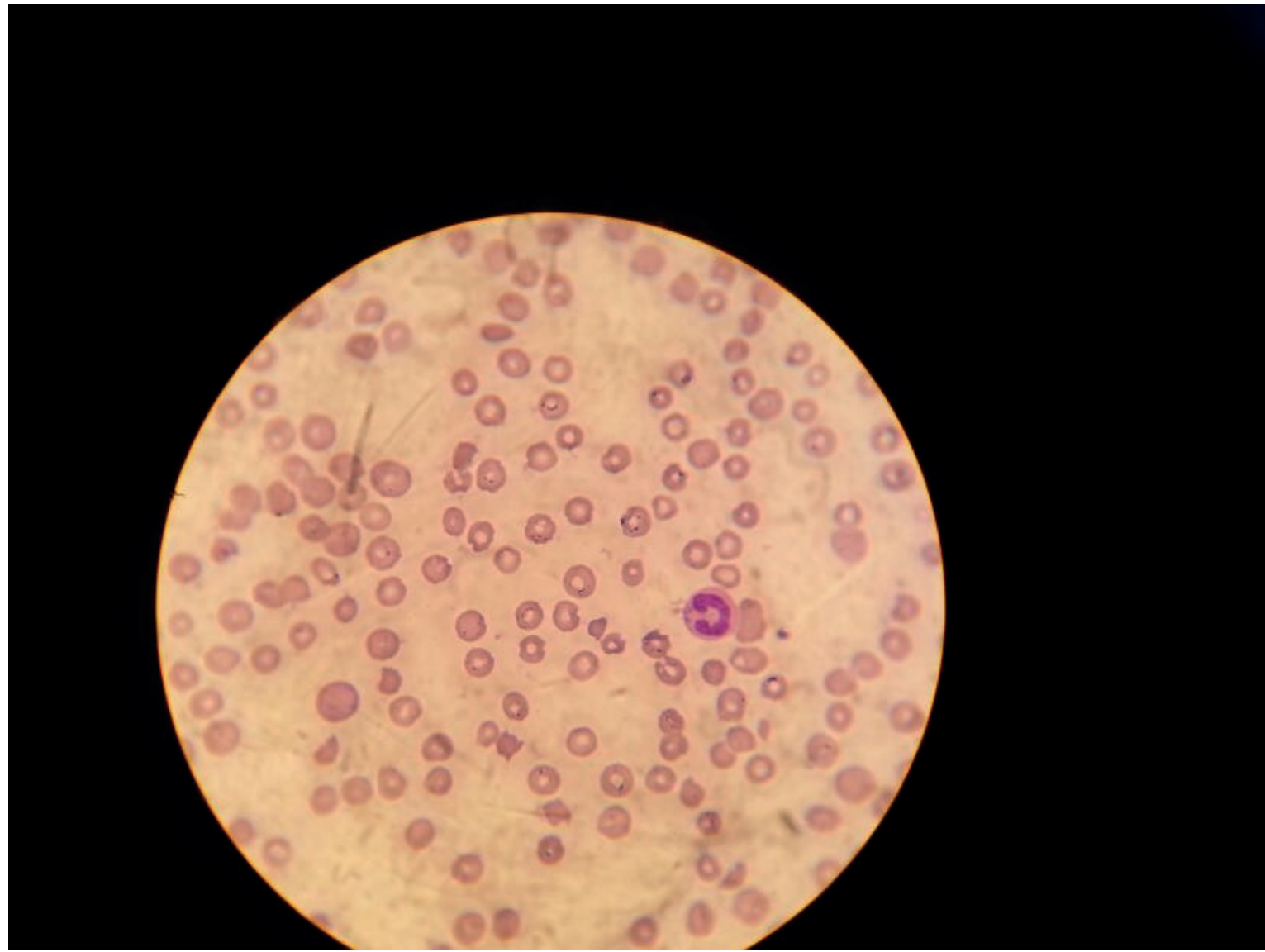

Fig. 1. Peripheral blood smear of the second case showing fragmented red cells (schistocytes), polychromatophilic red cells (reticulocytes), and a lack of platelets, consistent with the presence of microangiopathic hemolysis.

reticulocytes: 3.6\%, WBC: 16380/mm3, platelets: $4.000 / \mathrm{mm} 3$. Peripheral blood smear showed hypochromia, anisocytosis, poikilocytosis, polychromasia, and hemolysis along with occasional schistocytes and single platelets (Fig. 1). Direct Coombs test was negative. In biochemical evaluation the following were found; LDH: $1561 \mathrm{U} / \mathrm{L}$, total bilirubin: $1.4 \mathrm{~g} / \mathrm{dL}$, indirect bilirubin: $0.8 \mathrm{~g} /$ dL. Renal function was preserved. Urinalysis showed microscopic hematuria. Coagulation tests were normal, however D-dimer was $10,000 \mathrm{ng} / \mathrm{mL}$. Hemoglobin electrophoresis was normal.

The patient received platelets and red blood cells transfusion for anemia and thrombocytopenia. Bone marrow aspiration was performed for investigation of conditions that infiltrate the bone marrow. Leukemia was ruled out due to the presence of hemolysis and reticulocytosis, lack of any obvious blastic infiltration in the bone marrow, and lack of response to repeated platelet suspensions. Intravenous immunogloblin (IVIG) and pulse steroid treatments were given due to the persistence of severe thrombocytopenia as an immune etiology could not be excluded. Focus was placed on hemolysis related anemia and on thrombocytopenia causes. In order to test for the autoimmune disorders, Anti-dsDNA and antinuclear antibodies were requested, however the results were negative. C 3 and $\mathrm{C} 4$ levels were normal. Cold agglutination test was negative. Double negative $\mathrm{T}$ test performed for autoimmune lymphoproliferative diseases revealed negative results.

No immune causes could be demonstrated; research on nonimmune etiologies continued. The patient was treated with $20 \mathrm{ml} / \mathrm{kg}$ of fresh frozen plasma (FFP) therapy due to consideration of thrombotic microangiopathies. Normal renal function 
tests and blood pressure ruled out Hemolytic Uremic Syndrome (HUS), and normal coagulation parameters ruled out disseminated intravascular coagulation (DIC). Complement H, I, F levels and antibodies for atypical HUS, and ADAMTS-13 levels for TTP were sent. Prior to transfusion, viral serology revealed that CMV IgM: $37.3 \mathrm{U} / \mathrm{mL}$ (positive), CMV PCR of blood: 1440 copies / $\mathrm{mL}$ (positive), and urine: 11,600 copies / $\mathrm{mL}$ (positive). ADAMTS-13 activity was $<2 \%$ (40 to 130 ), antigen was $0.08 \mathrm{ug} / \mathrm{ml}(0.5-1.6)$, and no inhibitors were found. The patient responded to FFP replacement. The hemoglobin level increased to $8.4 \mathrm{~g} / \mathrm{dl}$ and a platelet count of $98 \times 10^{9} / 1$. In this patient, "Congenital TTP secondary to Cytomegalovirus Infection " was considered. Since the diagnosis was confirmed, he remained asymptomatic with a normal platelet count and did not require any other transfusion during his hospital stay. The family was informed about the disease and their consent was taken to publish this case. On the 50th day of diagnosis, thrombocytopenia recurred during an infectious episode. The patient's family declined further treatment, and the patient was lost to follow-up.

\section{Discussion}

Thrombotic thrombocytopenic purpura (TTP) was first described in 1924 by Moschowitz ${ }^{4}$ in an 8 year old girl patient in coma with anemia, fever, hematuria and petechiae. The classical pentad consists of hemolytic anemia, thrombocytopenia, renal failure, fuctuating neurological signs and fever.

The peak incidence of acquired TTP occurs between ages 30 and 40 . Children constitute $10 \%$ of the cases ${ }^{5}$. Twenty two percent of them may be diagnosed during infancy. Although the congenital form of the illness is expected to appear in early childhood, it may occur after age 18 in $26 \%$ of the patients, and it may remain asymptomatic even until beyond age $45^{6}$. In a study by Wang J et al. ${ }^{5}$ the age of onset of the congenital form ranged between the neonatal period and age 79. Therefore both forms of the illness demonstrate a wide age distribution within themselves. In concordance with previous reports, the disease in our patients manifested at different childhood periods, and with variable clinical presentations. The disease occurred in adolescence in the first patient, and infancy in the other.

The basic characteristics of the disease are hemolytic anemia and thrombocytopenia. Other characteristics such as renal, neurologic, and cardiac involvement due to ischemic organ injury are rarely seen in the congenital form. Neurologic symptoms such as headache, altered consciousness, seizures, paresis, and visual problems may be present in $67 \%$ of acquired TTP and $35 \%$ of congenital TTP patients. ${ }^{7}$ In TTP, minimal disruption in kidney functions with hematuria and proteinuria may accompany acute attacks. However acute renal failure generally occurs in the course of repeated episodes. In a study performed by Fujimara et al. ${ }^{8}$, among 43 patients diagnosed with congenital TTP, 2 patients had severe renal involvement. Similar to previous reports, our cases presented primarily with hemolytic anemia and thrombocytopenia, while renal and neurologic involvement were also present in the first patient who was an adolescent. Renal and neurologic functions were preserved in the second patient diagnosed with congenital TTP.

HUS is the most common condition that is misdiagnosed instead of TTP, and renal failure is helpful in the differentiation. In the classical form of HUS, there is an acute onset hemorrhagic diarrhea and acute renal failure caused by an infection due Escherichia coli secreting a shiga-like toxin. Although atypical HUS can develop without a previous history of bloody diarrhea, end stage renal failure develops in $50-80 \%$ of the cases and the mortality is high ${ }^{9}$. In contrast to HUS, severe renal involvement is not expected during the early period in TTP, however it may be added to the clinical picture late in the course. In the study by Tsai et al. ${ }^{10}$, among 9 patients diagnosed with congenital TTP, 5 developed progression toward end stage renal disease. A case report by Mise et al. ${ }^{11}$ published in 2013, presented a patient who was followed up from age 6 to 41 for HUS and end stage renal disease, and was later diagnosed with TTP at age 41 , by demonstration of the decrease in ADAMTS-13 activity and familial mutation. Because the clinical differentiation of these 2 conditions are very difficult due 
to their common properties, evaluation of ADAMTS-13 activity is more determinant. In the second patient HUS was ruled out by the initial absence of gastrointestinal complaints, lack of any findings which would suggest renal involvement during follow up such as hypertension, oliguria, renal test disorders, and finally by demonstration of the congenital absence of ADAMTS-13.

The diagnosis of congenital TTP is dependent on detecting ADAMTS13 activity $<5 \%$, in the absence of antibodies to ADAMTS13 and can be confirmed by demonstration of homozygous and heterozygous mutations in the ADAMTS-13 gene. Genetic studies have shown various mutations that affected different alleles; the variations in the clinical course of the disease were linked to the variety of these mutations. Although the molecular analysis couldn't be performed because of the technical reasons; the clinical and laboratory findings consistent with TTP, low ADAMTS-13 activity, the absence of antibodies and response to treatment supported the diagnosis of congenital TTP in the second patient without the need to perform a gene analysis study. İnheritance of congenital TTP has been thought to be autosomal recessive so a deficiency of ADAMTS-13 activity in firstdegree relatives especially in siblings is also a very strong indicator. Our patient was the only child in a family and no members had a history of thrombosis or bleeding episodes.

In a patient who has microangiopathic hemolytic anemia and thrombocytopenia, with signs of hemolysis on peripheral smear, there is no need to wait for the ADAMTS-13 test results to initiate treatment (plasmapheresis / FFP $)^{12}$. Beginning the treatment as soon as possible is the standart and required approach. Plasmapheresis, applied since 1980, has enabled a significant decrease especially in the mortality of acquired TTP. ${ }^{13}$ In acquired TTP plasma exchange is aimed to replace the deficient ADAMTS-13 and remove the autoantibodies. On the other hand, there are rare cases, as our first patient, in which plasmapheresis is not sufficient. During the management period of this patient, plasmapheresis was applied 18 times in combination with glucocorticoid treatment because it was the only appropriate treatment approach, however the patient did not benefit from it. Lately, immunosuppressive agents such as cyclosporine, rituximab, vincristine have emerged as promising approaches in the prevention of relapses and achieving remission in resistant cases but further studies are needed before these approaches can be universally recommended for children with TTP. ${ }^{12,14-16}$ In congenital TTP, the activity or quantity of the molecule has decreased due to the mutations in the ADAMTS-13 gene. In these patients, infusion of only FFP is sufficient, immunosuppressive treatment or splenectomy is not recommended. Our patient with congenital TTP had benefit from FFP replacement.

Although patients diagnosed with TTP generally show a good response to initial treatment, $40 \%$ of them experience a relapse within a mean period of 20 months. ${ }^{9}$ Some of the patients with congenital TTP require prophylactic plasma infusion every 3-4 weeks for replacing the functional ADAMTS-13 and preventing recurrent attacks. Some others are treated during conditions that trigger exacerbations, such as pregnancy or infection. Although the long term mortality rate of the disease is under $10 \%$, it reaches $90 \%$ without treatment. ${ }^{15,17}$

Although it is a rare underlying condition among pediatric patients who have predominantly signs of hemolytic anemia and thrombocytopenia, TTP should be considered in the differential diagnosis as it has a high mortality and morbidity rate. An early diagnosis and rapid appropriate treatment may decrease disease related sequela. A close follow up in the clinics is essential after discharge. In preventing relapses, it is important to select those patients who will receive prophylactic FFP. Risk and requirement should be evaluated, the treatment regimen should be individualized depending on the patient phenotype.

\section{Acknowledgements}

No author of this paper has a conflict of interest, including specific financial interest relationships and/or affiliations relevant to the subject matter or materials. 


\section{REFERENCES}

1. George JN. Thrombotic thrombocytopenic purpura. N Engl J Med 2006; 354: 1927-1935.

2. Borgi A, Khemiri M, Veyradier A, Kazdaghli K, Barsaoui S. Congenital thrombotic thrombocytopenic purpura: Atypical presentation and new ADAMTS 13 mutation in a Tunisian child. Mediterr J Hematol Infect Dis 2013; 5: e2013041.

3. Lotta LA, Garagiola I, Palla R, Cairo A, Peyvandi F. ADAMTS13 mutations and polymorphisms in congenital thrombotic thrombocytopenic purpura. Hum Mutat 2010; 31: 11-19.

4. Kremer Hovinga JA, Studt JD, Lämmle B. The von Willebrand factor-cleaving protease (ADAMTS-13) and the diagnosis of thrombotic thrombocytopenic purpura. Pathophysiol Haemost Thromb 2003/2004; 33: 417-421.

5. Wang J, Peiris N. Thrombotic thrombocytopenic purpura revisited: Upshaw-Schulman syndrome in a 20-year-old male. The Medicine Forum 2012; 13: 43-45.

6. Sarode R, Bandarenko N, Brecher ME, et al. Thrombotic thrombocytopenic purpura: 2012 American Society for Apheresis (ASFA) consensus conference on classification, diagnosis, management, and future research. J Clin Apher 2014; 29: 148-167.

7. Loirat C, Coppo P, Veyradier A. Thrombotic thrombocytopenic purpura in children. Curr Opin Pediatr 2013; 25: 216-224.

8. Fujimura $\mathrm{Y}$, Matsumoto M, Isonishi A, et al. Natural history of Upshaw-Schulman Syndrome based on ADAMTS13 gene analysis in Japan. J Thromb Haemost 2011; 9: (Suppl 1) 283-301.

9. Trachtman H. HUS and TTP in children. Pediatr Clin North Am 2013; 60: 1513-1526.
10. Tsai HM. The kidney in thrombotic thrombocytopenic purpura. Minerva Med 2007; 98: 731-747.

11. Mise K, Ubara $\mathrm{Y}$, Matsumoto $\mathrm{M}$, et al. Long term follow up of congenital thrombotic thrombocytopenic purpura (Upshaw-Schulman syndrome) on hemodialysis for 19 years: Acase report. BMC Nephrol 2013; 14: 156.

12. Jayabose S, Nowicki TS, Dunbar J, Levendoglu-Tugal O, Ozkaynak MF, Sandoval C. Acquired thrombotic thrombocytopenic purpura in children: A single institution experience. Indian J Pediatr 2013; 80: 570-575.

13. George JN. Congenital thrombotic thrombocytopenic purpura: Lessons for recognition and management of rare syndromes. Pediatr Blood Cancer 2008; 50: 947948.

14. Blombery P, Scully M. Management of thrombotic thrombocytopenic purpura: Current perspectives. J Blood Med 2014; 5: 15-23.

15. George JN, Al-Nouri ZL. Diagnostic and therapeutic challenges in the thrombotic thrombocytopenic purpura and hemolytic uremic syndromes. Hematology Am Soc Hematol Educ Program 2012; 2012: 604-609.

16. Appel GB. Thrombotic microangiopathies: Similar presentations, different therapies. Cleve Clin J Med 2017; 84: 114-130.

17. Scully M, Hunt BJ, Benjamin S, et al; British Committee for Standards in Haematology. Guidelines on the diagnosis and management of thrombotic thrombocytopenic purpura and other thrombotic microangiopathies. Br J Haematol 2012; 158: 323335. 